\title{
STRONG $n$-GENERATORS AND THE RANK OF SOME NOETHERIAN ONE-DIMENSIONAL INTEGRAL DOMAINS
}

\author{
KERSTIN PETTERSSON
}

\section{Introduction}

Throughout this paper any ring is a commutative Noetherian ring with identity. If $A$ is a ring, $I$ and $J$ are ideals of $A$ such that $I \supset J$, and $S$ is a multiplicative set of $A$ then let $\mu(I), \mu(I / J)$, and $\mu\left(S^{-1} I\right)$ respectively be the minimal number of generators of $I, I / J$, and $S^{-1} I$ respectively as an $A, A / J$, or $S^{-1} A$-module respectively.

Definition. Let $A$ be a ring and $n$ a positive integer. An ideal $I$ of $A$ is $n$ generated if $\mu(I) \leq n$, and if $\mu(I) \leq n$ for each ideal $I$ of $A$ then I.S. Cohen [6] says that $A$ is of finite rank $n$. S.T. Chapman and N.H. Vaughan [5] call a non-zero element $a$ in $A$ a strong $n$-generator if $a$ can be chosen as the first of $n$ generators of each ideal in which it is contained, and if moreover $a$ is contained in some ideal $I$ such that $\mu(I)=n$ then we introduce the concept proper strong $n$-generator for $a$. An ideal $I$ of $A$ is strongly $n$-generated if $I$ is $n$-generated and $\mu(I /(a))<n$ for each non-zero element $a$ of $I$.

Notation. If $A$ is an integral domain then let $\bar{A}$ be the integral closure of $A$, and if $A$ is of finite rank then let $\mu_{*}(A)=\max \{\mu(I): I$ is an ideal of $A\}$. Let $R$ be a one-dimensional integral domain, let $F$ be the field of fractions of $R$, and let $C$ be the conductor of $R$ in $\bar{R}$, i.e. $C=(R: \bar{R})_{R}$. If $I$ is a non-zero ideal of $R$ then $\operatorname{Ass}(I)$ is the set of maximal ideals that contain $I$.

We have that $\bar{R}$ is an integrally closed one-dimensional domain, and hence $\bar{R}$ is a Dedekind domain. It is well-known that a Dedekind domain is strongly two-generated, and we also know that $\operatorname{Ass}(I)$ is finite for each nonzero ideal $I$ of $R$ since $R$ is Noetherian.

Let $C$ be non-zero which is the same as $\bar{R}$ being a fractional ideal of $R$, and hence $\bar{R}$ is a finitely generated $R$-module. By I.S. Cohen [6], Theorem 10 and Corollary $3, R$ is of finite rank, and if $\mu_{*}\left(R_{M}\right) \leq n-1$ for each maximal

Received March 5, 1997. 
ideal $M$ of $R$ then $\mu_{*}(R) \leq n$ for some integer $n$. By H. Bass [4], Proposition $1.4, \mu_{*}(R) \leq \max (2, k)$ if $\mu_{*}\left(R_{M}\right) \leq k$ for each maximal ideal $M$ of $R$. A specification of these results is that if $R$ is not integrally closed then $\mu_{*}(R)=\mu(C)=\mu\left(C_{M}\right)=\mu_{*}\left(R_{M}\right)$ for some $M \in \operatorname{Ass}(C)$ (Theorem 15). In Proposition 3 is proved that an ideal $I$ of $R$ is strongly $\mu(I)$-generated if and only if $\mu(I)=2$, and $\mu\left(I / I^{2}\right)=1$. Let $M \in \operatorname{Ass}(C)$. Then there is a unique biggest $M$-primary ideal $I$ such that $\mu(I)=\mu_{*}\left(R_{M}\right)$ (Proposition 16), and hence if $M$ is such that $\mu_{*}\left(R_{M}\right)=\mu_{*}(R)$ then $\mu(I)=\mu_{*}(R)$. In [12], Theorem 21 we have already proved that the set of strong $\mu_{*}(R)$-generators of $R$ is $R \backslash \bigcup_{i \in S}\left(I_{i} M_{i}\right)$ where $\left\{M_{i}\right\}_{i \in S}$ is the set of maximal ideals of $R$ such that $\mu_{*}\left(R_{M_{i}}\right)=\mu_{*}(R)$, and $I_{i}$ is the unique biggest $M_{i}$-primary ideal of $R$ such that $\mu\left(I_{i}\right)=\mu_{*}(R)$. Let $P$ be the set of proper strong $\mu_{*}(R)$-generators of $R$. If $\mu_{*}(R)=2$, and $\left\{M_{i}\right\}_{i \in T}$ is the set of maximal ideals of $R$ such that $M_{i} \notin \operatorname{Ass}(C)$, and $M_{i} \bar{R} \neq r \bar{R}$ for any $r \in R$ then $P=\bigcup_{M \in A s s(C)}\left(M \backslash M^{2}\right) \bigcup_{i \in T}$ $M_{i}$ (Theorem 11). If $\mu_{*}(R)>2$ then the set $\left\{M_{i}\right\}_{i \in S}$ of maximal ideals of $R$ such that $\mu_{*}\left(R_{M_{i}}\right)=\mu_{*}(R)$ is finite and contained in $\operatorname{Ass}(C)$. If $S=\{1, \ldots, k\}$ then $P=\bigcup_{i=1}^{k}\left(I_{i} \backslash I_{i} M_{i}\right)$, where $I_{i}$ is the unique biggest $M_{i^{-}}$ primary ideal of $R$ such that $\mu\left(I_{i}\right)=\mu_{*}(R), i=1, \ldots, k$ (Theorem 18). In Section 4 we treat rings strictly between $K$ and $K[X]$ where $K$ is a field. In Proposition 19 we prove that any such ring has non-zero conductor in its integral closure. If $R$ is a ring such that $K\left[X^{k}\right] \subseteq R \subseteq K[X]$ where $k$ is a positive integer, and $R \nsubseteq K\left[X^{l}\right]$ for any $l>1$ then the field of fractions of $R$ is $K(X)$ (Lemma 20), the integral closure of $R$ is $K[X]$, and $\mu_{*}(R) \leq k$ (Proposition 21). For the special case $R=K\left[X^{k}, a X^{l}\right]$ with $a$ in $K\left[X^{k}\right] \backslash K$ and some $k, l \in \mathrm{N}$ such that $(k, l)=1$ we show that $\mu_{*}(R)=k$, and for each maximal ideal $M$ such that $\mu_{*}\left(R_{M}\right)=k, M^{k-1}$ is the biggest $M$-primary ideal $I$ such that $\mu(I)=k$ (Proposition 23). Let $n_{1}, \ldots, n_{l}$ be positive integers such that $\left(n_{1}, \ldots, n_{l}\right)=1$, and let $N$ be the least integer such that for any $i \geq 0, N+i$ belongs to the numerical semigroup generated by $\left\{n_{1}, \ldots, n_{l}\right\}$. If the numerical semigroup ring $K\left[X^{n_{1}}, \ldots, X^{n_{l}}\right]$ is contained in $R$, and $R$ is contained in $K[X]$ then $C=X^{N} K[X]$, and if $M=X K[X] \cap R$ then $\mu_{*}(R)=\min v(M)$ where $v: K(X) \longrightarrow \mathrm{Z}$ is the valuation of $K(X)$ with $K[X]_{(X)}$ as valuation ring (Proposition 24).

\section{Preliminaries}

Let $A$ be a domain, let $I$ be a non-zero ideal of $A$, and let $S_{I}=A \backslash \bigcup_{i \in S} M_{i}$ where $\left\{M_{i}\right\}_{i \in S}$ is the set of maximal ideals of $A$ which contain $I$.

Proposition 1. Let $A$ be a one-dimensional domain, and let I be an ideal of A such that $\mu(I)>2$. Then $\mu\left(I_{M}\right)=\mu\left(S_{I}^{-1} I\right)=\mu(I)$ for some $M \in \operatorname{Ass}(I)$. 
Proof. We have $\mu\left(I_{M}\right) \leq \mu(I)$ for each maximal ideal $M$ of $A$. By [8], Satz $1, \mu(I) \leq \max \left\{\mu\left(I_{p}\right)+\operatorname{dim} A / p: p \in \operatorname{Spec} A\right\} \leq \max \left\{\mu\left(I_{M}\right), 2: M \in\right.$ $\operatorname{Ass}(I)\} \leq \mu(I)$ whence $\mu(I)=\mu\left(S_{I}^{-1} I\right)=\mu\left(I_{M}\right)$ for some $M \in \operatorname{Ass}(I)$.

Lemma 2. Let $A$ be an integral domain with non-zero conductor $C$ in the integral closure $\bar{A}$ of $A$. If $s$ is comaximal to $C, a \in \bar{A}$, and sa $A$ then $a \in A$.

Proof. We have $s b=1+r$ for some $r \in C$, and some $b \in A$. Then $a s b=a+r a \in A$, and hence $a \in A$.

\section{One-dimensional integral domains}

Let $R$ be a one-dimensional integral domain with field of fractions $F$, and let the conductor $C$ of $R$ in $\bar{R}$ be non-zero. If $M$ is a maximal ideal of $R$ then $\overline{R_{M}}=(R \backslash M)^{-1} \bar{R}$ by [2], Proposition 5.12.

Proposition 3. Let $I$ be an ideal of $R$. Then the following are equivalent:

1) I is strongly $\mu(I)$-generated.

2) $\mu(I)=2$, and $\mu\left(S_{I}^{-1} I\right)=1$.

3) $\mu(I)=2$, and $\mu\left(I / I^{2}\right)=1$.

4) $\mu(I)=2$, and $\mu\left(I_{M}\right)=1$ for each maximal ideal $M$ of $R$.

Proof. 1) $\Rightarrow 2$ ) Let $I$ be an ideal of $R$ which is strongly $\mu(I)$-generated. By [1], Theorem $8, \mu\left(S_{I}^{-1} I\right)<\mu(I)$, and hence $\mu(I)=2$ by Proposition 1 , and $\mu\left(S_{I}^{-1} I\right)=1$.

2) $\Leftrightarrow 3)$ By [1], Proposition $1, \mu\left(I / I^{2}\right)=\mu\left(S_{I}^{-1} I\right)$.

2) $\Rightarrow 4$ ) is obvious.

$4) \Rightarrow 1$ ) By [12], Proposition 19, I is strongly two-generated.

Lemma 4. Let $M$ be a maximal ideal of $R$ such that $M \notin \operatorname{Ass}(C)$. Then there is a unique maximal ideal $N$ of $\bar{R}$ such that $N \cap R=M$. Moreover $M \bar{R}=N$, and $\overline{R_{M}}=R_{M}$ is a discrete valuation ring (DVR).

Proof. By [14], Ch. 5, §5, Lemma, $R_{M}=\overline{R_{M}}$. By [2], Proposition 9.2, $R_{M}$ is a DVR, and by [2], Proposition 3.11, $M R_{M}=N \overline{R_{M}}$ for some unique maximal ideal $N$ of $\bar{R}$. Then $N \cap R=M$, and by [2], Proposition 1.14, $M \bar{R}$ is $N$-primary. Since $\bar{R}$ is a Dedekind domain $M \bar{R}=N^{l}$ for some $l \in \mathrm{N}$. But $N \overline{R_{M}}=M R_{M}=M \overline{R_{M}}=N^{l} \overline{R_{M}}$, and hence $l=1$.

COROLlary 5. Let $I$ be an ideal of $R$ such that $\mu(I)>2$. Then $\mu\left(I_{M}\right)=\mu(I)$ for some $M \in A \operatorname{ss}(C)$.

Proof. The result follows from Proposition 1 and the previous lemma.

If $R \neq \bar{R}, \mu(I)=2$, and $I$ is not comaximal to $C$ then we see in the fol- 
lowing example that $\mu\left(S_{I}^{-1} I\right)$ is not in general equal to $\mu(I)$, and $\mu\left(I_{M}\right)$ can be less than $\mu(I)$ for each $M \in \operatorname{Ass}(C)$ whence $I$ is strongly $\mu(I)$-generated by Proposition 3 .

Example. Let $R=K\left[X^{2}, X^{3}\left(1+X^{2}\right)\right]$, let $M_{1}=\left(X^{2}, X^{3}\left(1+X^{2}\right)\right)$, $M_{2}=\left(1+X^{2}, X^{3}\left(1+X^{2}\right)\right)$, and let $I=\left(X^{3}\left(1+X^{2}\right),\left(1+X^{2}\right)^{2}\right)$. Then $\mu(I)=2, C=X^{2}\left(1+X^{2}\right)(1, X)$ (see Proposition 22), Ass $(C)=\left\{M_{1}, M_{2}\right\}$, and $I$ is $M_{2}$-primary. Hence $I_{M_{1}}=R_{M_{1}}$, and $S_{I}^{-1} I=I_{M_{2}}$. Since $\left(1+X^{2}\right)^{2}=\left(X^{3}\left(1+X^{2}\right)\right)^{2} / X^{6}$ we have $\mu\left(I_{M_{2}}\right)=1$.

E.D. Davis and A.V. Geramita have proved in [7], Theorem 1 that $\mu(M)=\mu\left(M_{M}\right)$ if $R_{M}$ is not regular, i.e. if $R_{M}$ is not a DVR.

Lemma 6. Suppose $R \neq \bar{R}$. If $M \in \operatorname{Ass}(C)$ then $\mu(M)=\mu\left(M_{M}\right) \geq 2$, and $M$ is not strongly $\mu(M)$-generated. Moreover $\mu_{*}(R)=2$ if and only if $\mu_{*}\left(R_{M}\right)=2$ for each $M \in \operatorname{Ass}(C)$.

Proof. Suppose $M \in \operatorname{Ass}(C)$. If $M$ is principal then $M_{M}$ is principal, and by [2], Proposition 9.2, $R_{M}$ is integrally closed. By [14], Ch. 5, $\S 5$, Lemma, $C \nsubseteq \mathbb{M}$ which is a contradiction. Thus $\mu(M) \geq \mu\left(M_{M}\right) \geq 2$. By Proposition 1, $\mu(M)=\mu\left(M_{M}\right)$, and by Proposition 3, $M$ is not strongly $\mu(M)$-generated. The last statement is true since $2 \leq \mu_{*}\left(R_{M}\right) \leq \mu_{*}(R)$.

Lemma 7. Let $I$ be an ideal of $R$ which is comaximal to $C$. Then $I$ is a product of maximal ideals.

Proof. By [2], Proposition 9.1, $I=\prod_{i=1}^{l} q_{i}$ where $q_{i}$ is $M_{i}$-primary for $i=1, \ldots, l$, and $M_{i} \neq M_{j}$ if $i \neq j$. By Lemma $4, R_{M_{i}}$ is a DVR, and by [2], Proposition 9.2, $q_{i_{M_{i}}}=M_{i M_{i}}^{k_{i}}$ for some $k_{i} \in \mathrm{N}, i=1, \ldots, l$. By [2], Propositions 3.11 and $4.8, q_{i}=M_{i}^{k_{i}}, i=1, \ldots, l$.

Lemma 8. Let $I$ be an ideal of $R$ which is comaximal to $C$. Then $I=I \bar{R} \cap R$.

Proof. By Lemma 7, I $\bar{R} \cap R=\left(\prod_{i=1}^{l} M_{i}^{k_{i}}\right) \bar{R} \cap R=\left(\prod_{i=1}^{l}\left(M_{i} \bar{R}\right)^{k_{i}}\right) \cap R$. By Lemma 4, $I \bar{R} \cap R=\bigcap_{i=1}^{l}\left(M_{i} \bar{R}\right)^{k_{i}} \cap R \subseteq \bigcap_{i=1}^{l}\left(\left(M_{i} R_{M_{i}}\right)^{k_{i}} \cap R\right)$ and by [2], Proposition 3.11 and 4.8, $I \bar{R} \cap R \subseteq \bigcap_{i=1}^{l} M_{i}^{k_{i}}=I$. Hence $I \bar{R} \cap R=I$.

Proposition 9. Let I be an ideal of $R$ which is comaximal to $C$. Then $I$ is strongly two-generated.

Proof. If $M \in \operatorname{Ass}(C)$ then $I_{M}=R_{M}$, and if $M \notin \operatorname{Ass}(C)$ then by Lemma $4, R_{M}$ is a DVR. Hence $I_{M}$ is principal for each maximal ideal $M$ of $R$, and by [12], Proposition 19, $I$ is strongly two-generated.

Lemma 10. Let $I$ be an ideal of $R$ which is comaximal to $C$. Then $I$ is principal if and only if there is $r \in R$ such that $I \bar{R}=r \bar{R}$. 
Proof. $\Rightarrow)$ Suppose $I$ is principal. Then $I=(r)$ for some $r \in R$. Hence $I \bar{R}=r \bar{R}$.

$\Leftrightarrow)$ Assume there is $r \in R$ such that $I \bar{R}=r \bar{R}$. By Proposition 9, $I=(s, t)$ for some $s, t \in R$, and $(s, t) \bar{R}=r \bar{R}$. Hence $s=u r$ and $t=v r$ for some $u, v \in \bar{R}$. Since $r \bar{R} \nsubseteq \subset M \bar{R}$ for any $M \in \operatorname{Ass}(C)$ we have that $r$ is comaximal to $C$, and by Lemma $2, u, v \in R$, and thus $(u, v)$ is comaximal to $C$. By Lemma $8,(u, v)=(u, v) \bar{R} \cap R=R$, and thus $I$ is principal.

THEOREM 11. Let $R$ be a one-dimensional domain with non-zero conductor $C$ in the integral closure $\bar{R}$ of $R$. Let $\mu_{*}(R)=2$, and let $\left\{M_{i}\right\}_{i \in S}$ be the maximal ideals of $R$ such that $M_{i} \notin \operatorname{Ass}(C)$, and $M_{i} \bar{R} \neq r \bar{R}$ for any $r \in R$ and $i \in S$. Then the set of proper strong two-generators of $R$ is $\bigcup_{M \in \operatorname{Ass}(C)}\left(M \backslash M^{2}\right) \bigcup_{i \in S} M_{i}$.

Proof. Let $I$ be an ideal of $R$ such that $\mu(I)=2$. If $I$ is not comaximal to $C$ then $I \subseteq M$ for some $M \in \operatorname{Ass}(C), R \neq \bar{R}$ and $\mu(M)=\mu\left(M_{M}\right)=2$ by Lemma 6. If $I$ is comaximal to $C$ then $I$ is a product of maximal ideals by Lemma 7, and hence there is a non-principal maximal ideal $M$ which contains $I$ and is comaximal to $C$. By Lemma $10, M \in\left\{M_{i}\right\}_{i \in S}$. By [12], Theorem 21 the set of proper strong two-generators of $R$ is $\bigcup_{M \in \operatorname{Ass}(C)}\left(M \backslash M^{2}\right) \bigcup_{i \in S} M_{i}$.

Definition. Let $(A, M)$ be a one-dimensional local Noetherian domain, let $I$ be an ideal of $A$, and let $\ell(B)$ be the length of a finitely generated $A$ module $B$. Then $C$. Gottlieb [9] calls $I$ a maximally generated ideal if $\mu(I)=\min \{\ell(A /(a)): a \in M\}$ or equivalently $M I=a I$ for some $a \in M$, and then $\mu(I)=\mu_{*}(A)$. An element $a$ of $M$ is superficial if there exists a positive integer $c$ such that $\left(M^{l}: a\right) \cap M^{c}=M^{l-1}$ for any sufficiently large integer $l$ cf. [14], p. 285.

Remark 12. If $(A, M)$ is a one-dimensional local Noetherian domain then $A$ is Cohen-Macaulay. Suppose $a$ is a superficial element of $M$. By definition $a^{-1} M^{l} \cap M^{c}=M^{l-1}$, and thus $a M^{l-1}=a\left(a^{-1} M^{l} \cap M^{c}\right)=M^{l} \cap a M^{c}$. Since $a$ is $M$-primary, $M^{c+i} \subseteq a M^{c}$ for some positive integers $c, i$, and hence $a M^{k}=M^{k+1}$ for each integer $k$ which is large enough. Then $M^{k}$ is a maximally generated ideal, and $\min \{\ell(A /(b)): b \in M\}=\ell(A /(a))=\mu\left(M^{k}\right)=$ $\mu_{*}(A)$. By [10], Theorems 14.13 and 17.11, $e(A)=e(M, A)=e((a), A)=$ $\ell(A /(a))=\mu\left(M^{k}\right)$ where $e(A)$ is the multiplicity of $A$. If $A$ has infinite residue field then $A$ has a superficial element by [14], p. 287.

Lemma 13. Let $(A, M)$ be a one-dimensional local domain with a superficial element s. Let $\bar{A}$ be the integral closure of $A$, and let $C$ be the non-zero conductor of $A$ in $\bar{A}$. Then $C$ is a maximally generated ideal of $A$.

Proof. By Remark 12, $s M^{k}=M^{k+1}$ for some $k \in \mathrm{N}$, and hence 
$s \bar{A}(M \bar{A})^{k}=s M^{k} \bar{A}=M^{k+1} \bar{A}=(M \bar{A})^{k+1}$. Because of unique factorization of an ideal in $\bar{A}$ as a product of prime ideals we have $s \bar{A}=M \bar{A}$. Since $C$ is an ideal of $\bar{A}$ we have $M C=M C \bar{A}=s C \bar{A}=s C$, and $C$ is maximally generated by definition.

Notation. If $M$ is a maximal ideal of $R$ then let $R_{M}(u)=R[u]_{M[u]}$.

REMARK 14. Since $R_{M}(u)$ is CM and has infinite residue field, $R_{M}(u)$ has a superficial element and a maximally generated ideal by Remark 12 . If $I$ is an ideal of $R$ then $\mu\left(I R_{M}(u)\right)=\ell_{R_{M}(u)}\left(I R_{M}(u) / I M R_{M}(u)\right)=\ell_{R_{M}}\left(I_{M} / I_{M} M_{M}\right)=$ $\mu\left(I_{M}\right)$, and since any ideal of $R_{M}(u)$ is an extended ideal of $R_{M}$, $\mu_{*}\left(R_{M}(u)\right)=\mu_{*}\left(R_{M}\right)$. By [10], Theorem $12.4, \overline{R_{M}}[u]$ is integrally closed, and hence $\overline{R_{M}}(u)$ is also. The integral closure of $R_{M}[u]$ in $F[u]$ is $\overline{R_{M}}[u]$ by [2], Ch. 5, Exercise 9, and thus $\overline{R_{M}[u]}=\overline{R_{M}}[u]$, and by [2], Proposition 5.12, $\overline{R_{M}(u)}=(R[u] \backslash M R[u])^{-1} \overline{R_{M}}[u]$.

THEOREM 15. Let $R$ be a one-dimensional domain with non-zero conductor $C$ in the integral closure $\bar{R}$ of $R$, and let $R \neq \bar{R}$. Then $\mu_{*}(R)=\mu(C)=$ $\mu\left(C_{M}\right)=\mu_{*}\left(R_{M}\right)$ for some $M \in \operatorname{Ass}(C)$. If moreover $\bar{R}$ is a principal ideal domain (PID) then $\mu_{*}(R)=\mu(\bar{R})$ where $\bar{R}$ is considered as a fractional ideal of $R$.

Proof. Let $M \in \operatorname{Ass}(C)$. By [14], Ch. 5, §5, Lemma, $C_{M}$ is the conductor of $R_{M}$ in $\overline{R_{M}}$. Obviously $C R_{M}[u]$ is contained in the conductor of $R_{M}[u]$ in $\overline{R_{M}[u]}$. If $b \notin C R_{M}[u]$ then there is $a \in \overline{R_{M}}$ such that $a b \notin R_{M}[u]$. Hence $C R_{M}[u]$ is the conductor of $R_{M}[u]$ in $\overline{R_{M}[u]}$, and by [14], Ch. 5, $\S 5$, Lemma, the conductor of $R_{M}(u)$ in $\overline{R_{M}(u)}$ is $C R_{M}(u)$. By Remark 14 there is a superficial element $s$ in $R_{M}(u)$, and by Lemma $13, C R_{M}(u)$ is a maximally generated ideal of $R_{M}(u)$. By Remark 14, $\mu(C) \geq \mu\left(C_{M}\right)=\mu\left(C R_{M}(u)\right)=$ $\mu_{*}\left(R_{M}(u)\right)=\mu_{*}\left(R_{M}\right)$. Let $I$ be an ideal of $R$ such that $\mu(I)=\mu_{*}(R)>2$. By Corollary 5, $\mu(I)=\mu\left(I_{M^{\prime}}\right)$ for some $M^{\prime} \in \operatorname{Ass}(C)$, and hence $\mu_{*}(R)=$ $\mu_{*}\left(R_{M^{\prime}}\right)$ for some $M^{\prime \prime} \in \operatorname{Ass}(C)$. If $\mu_{*}(R)=2$ then $\mu_{*}\left(R_{M^{\prime}}\right)=2$ for any $M^{\prime} \in \operatorname{Ass}(C)$ by Lemma 6 . Let $M$ be such that $\mu_{*}\left(R_{M}\right)=\mu_{*}(R)$. Then $\mu(C)=\mu\left(C_{M}\right)=\mu_{*}\left(R_{M}\right)=\mu_{*}(R)$. If $\bar{R}$ is a PID then $C=c \bar{R}$ for some $c \in R$, and hence $\mu(C)=\mu(\bar{R})$ if $\bar{R}$ is considered as a fractional ideal of $R$.

Proposition 16. Let $R \neq \bar{R}$ and $M \in \operatorname{Ass}(C)$. Then there is a unique biggest $M$-primary ideal I of $R$ such that $\mu(I)=\mu_{*}\left(R_{M}\right)$.

Proof. Let $\mu_{*}(R)>2$, and let $I_{M}$ and $J_{M}$ be maximal among ideals of $R_{M}$ such that $\mu\left(I_{M}\right)=\mu\left(J_{M}\right)=\mu_{*}\left(R_{M}\right)$. By Remark 14, $\mu\left(I R_{M}(u)\right)=$ $\mu\left(J R_{M}(u)\right)=\mu_{*}\left(R_{M}(u)\right)$, and $I R_{M}(u)$ and $J R_{M}(u)$ are maximally generated ideals of $R_{M}(u)$. By [9], Lemma 4, $\mu\left(I R_{M}(u)+J R_{M}(u)\right)=\mu_{*}\left(R_{M}(u)\right)$, and by Remark 14, $\mu\left(I_{M}+J_{M}\right)=\mu_{*}\left(R_{M}\right)$ whence $I_{M}=J_{M}$, and $I_{M}$ is the unique 
biggest ideal of $R_{M}$ such that $\mu\left(I_{M}\right)=\mu_{*}\left(R_{M}\right)$. Let $I$ be the ideal of $R$ such that $I=I_{M} \cap R$. Then $I$ is $M$-primary, and $S_{I}^{-1} I=I_{M}$. By Lemma 6 , $\mu(I) \geq \mu\left(I_{M}\right)=\mu_{*}\left(R_{M}\right) \geq 2$, and by Proposition 1, $\mu(I)=\mu\left(I_{M}\right)=\mu_{*}\left(R_{M}\right)$. Let $q$ be an $M$-primary ideal of $R$ such that $\mu(q)=\mu_{*}\left(R_{M}\right)$. If $\mu_{*}\left(R_{M}\right)=2$ then $\mu\left(M_{M}\right)=2$ by Lemma 6 , and $q \subseteq M=I$. If $\mu_{*}\left(R_{M}\right)>2$ then $\mu\left(q_{M}\right)=\mu(q)$ by Proposition 1 , and hence $q_{M} \subseteq I_{M}$, whence $q \subseteq I$. If $\mu_{*}(R)=2$ then $\mu(M)=\mu_{*}\left(R_{M}\right)$ by Lemma 6 .

Remark 17. Let $R \neq \bar{R}$ and $M \in \operatorname{Ass}(C)$. If $\left(\overline{R_{M}}, m\right)$ is local, and $\overline{R_{M}} / m \simeq$ $R_{M} / M_{M}$ then let $I$ be the biggest maximally generated ideal of $R_{M}(u), s$ a superficial element of $R_{M}(u)$, and $J$ a fractional ideal of $R_{M}(u)$ which fulfils $M_{M}(u)=(s)+s J$ and such that $J \subseteq \overline{R_{M}(u)}$. Then by [3], Corollary 4, $I=\left(\ldots\left((C: J)_{R_{M}(u)}: J\right)_{R_{M}(u)} \ldots: J\right)_{R_{M}(u)}$, and $I^{\prime}=I \cap R$ is the biggest $M$ primary ideal of $R$ such that $\mu\left(I^{\prime}\right)=\mu_{*}\left(R_{M}\right)$. If there is a superficial element in $R_{M}$, and $\left(\overline{R_{M}}, m\right)$ is as above then the biggest maximally generated ideal of $R_{M}$ can be determined by [3], Corollary 4 .

THeOREM 18. Let $R$ be a one-dimensional domain with non-zero conductor $C$ in the integral closure $\bar{R}$ of $R$. Let $\mu_{*}(R)>2$, let $\left\{M_{i}\right\}_{i=1}^{k}$ be the set of maximal ideals of $R$ such that $\mu_{*}\left(R_{M_{i}}\right)=\mu_{*}(R)$. Then there is a unique biggest $M_{i}$-primary ideal $I_{i}$ of $R$ such that $\mu\left(I_{i}\right)=\mu_{*}(R), i=1, \ldots, k,\left\{M_{i}\right\}_{i=1}^{k} \subseteq \operatorname{Ass}(C)$, and the set of proper strong $\mu_{*}(R)$-generators of $R$ is $\bigcup_{i=1}^{k}\left(I_{i} \backslash I_{i} M_{i}\right)$.

Proof. Since $\mu_{*}(R)>2$ we have $R \neq \bar{R}$. By Theorem $15, R$ has finite rank. By Lemma $4, M_{i} \in \operatorname{Ass}(C), i=1, \ldots, k$. By Proposition 16 there is a unique biggest $M_{i}$-primary ideal $I_{i}$ of $R$ such that $\mu\left(I_{i}\right)=\mu_{*}\left(R_{M_{i}}\right)=\mu_{*}(R)$, $i=1, \ldots, k$. Let $I$ be an ideal of $R$ such that $\mu(I)=\mu_{*}(R)$. By Corollary 5 , $\mu\left(I_{M_{i}}\right)=\mu_{*}(R)$, and $I_{M_{i}} \cap R$ is $M_{i}$-primary for some $i \in\{1, \ldots, k\}$. Since $\mu\left(I_{M_{i}} \cap R\right) \geq \mu\left(\left(I_{M_{i}} \cap R\right)_{M_{i}}\right)=\mu\left(I_{M_{i}}\right)=\mu_{*}(R)$ we have $I_{i} \supseteq I_{M_{i}} \cap R \supseteq I$. By [12], Theorem 21, the set of proper strong $\mu_{*}(R)$-generators is $\bigcup_{i=1}^{k}\left(I_{i} \backslash I_{i} M_{i}\right)$.

\section{Integral domains contained in $K[X]$}

Let $K$ be a field, and let $R$ be a subring of $K[X]$ which strictly contains $K$. Then $K[X]$ is integral over $R$, and hence $R$ is a one-dimensional domain. By the next proposition $R$ has non-zero conductor in its integral closure.

Notation. Let $v: K(X) \longrightarrow \mathbf{Z}$ be the valuation of $K(X)$ with $K[X]_{(X)}$ as the valuation ring.

From Lemma 20 on, the special case when $X^{k} \in R$ for some positive integer $k$ is treated, and in Proposition 24, $X^{l} \in R$ for each sufficiently large integer $l$, i.e. $R$ contains the semigroup ring $K\left[X^{n_{1}}, \ldots, X^{n_{t}}\right]$ for some positive 
integers $n_{1}, \ldots, n_{t}$ such that $\left(n_{1}, \ldots, n_{t}\right)=1$. In that case we show that $\mu_{*}(R)=\min v(M)$ where $M=X K[X] \cap R$, and the conductor of $R$ in $K[X]$ is equal to $X^{N} K[X]$ where $N$ is the least integer such that $X^{N+i} \in R, i=0,1, \ldots$ The notation of Section 3 are used throughout this section.

Proposition 19. Let $K$ be a field, let $R$ be a subring of $K[X]$ which strictly contains $K$, and let $F$ be the field of fractions of $R$. Then the conductor $C$ of $R$ in its integral closure $\bar{R}$ is non-zero, and $\bar{R}=F \cap K[X]$.

Proof. Since $X$ is integral over $R, R[X]=K[X]$ is a finitely generated $R$ module, and hence $K[X]$ is a Noetherian $R$-module. By [2], Proposition 7.8, $R$ is Noetherian. Since $K[X]$ is integrally closed, and $F \subseteq K(X)$ we have that $\bar{R} \subseteq K[X]$. Therefore $\bar{R}=F \cap K[X]$ is a finitely generated $R$-module, and hence $C \neq 0$.

Lemma 20. Let $K$ be a field, and let $k$ be a positive integer. If $R$ is such that $K\left[X^{k}\right] \subseteq R \subseteq K[X]$, and $R \nsubseteq K\left[X^{l}\right]$ for any $l>1$ then $F=K(X)$.

Proof. We have that $K(X)$ contains $F$, and $F$ strictly contains $K\left(X^{k}\right)$. The minimum polynomial of $X$ over $K\left(X^{k}\right)$ is $t^{k}-X^{k}$. By [13], Theorem 3.2 the minimum polynomial $m$ of $X$ over $F$ divides $t^{k}-X^{k}$ in $F$. By [13], Theorem 4.2 and Proposition 4.3 the degree of $m$ is less than $k$. Let $r$ be the degree of $m$, and let $G$ be a splitting field of $m$. Then the zeros of $m$ in $G$ are $g_{1} X, \ldots, g_{r} X$ for some non-zero $g_{1}, \ldots, g_{r} \in G$, and hence the constant term of $m$ is $(-1)^{r} \prod_{i=1}^{r} g_{i} X^{r}$ where $\prod_{i=1}^{r} g_{i}$ is non-zero. Then $K\left(X^{r}\right) \subseteq F$, and by induction $K(X) \subseteq F$.

Proposition 21. Let $K$ be any field, let $k$ be a positive integer, and let $R$ be a ring such that $K\left[X^{k}\right] \subseteq R \subseteq K[X], X^{l} \notin R$ if $l<k$, and $R \nsubseteq K\left[X^{l}\right]$ for any $l>1$.

1) Then the integral closure of $R$ is $K[X]$, and $\mu_{*}(R)=\mu(K[X]) \leq k$ where $K[X]$ is considered as a fractional ideal of $R$.

2) If $\sum_{i=1}^{k-1} a_{i} X^{i} \in R$ for some $a_{i} \in K\left[X^{k}\right], i=1, \ldots, k-1$, and $a_{j} \in K \backslash\{0\}$ for some $j \in\{1, \ldots, k-1\}$ then $\mu_{*}(R)<k$.

Proof. 1) By Proposition 19 and Lemma 20, $K[X]$ is the integral closure of $R$. As $K[X]=\left(1, X, \ldots, X^{k-1}\right) R$ we have that $\mu(K[X]) \leq k$. By Theorem $15, \mu_{*}(R)=\mu(K[X])$.

2) We have $K[X]=\left(1, X, \ldots, X^{k-1}\right) R$, and by assumption $X^{j} \in(1, X, \ldots$, $\left.X^{j-1}, X^{j+1}, \ldots, X^{k-1}\right) R$. Hence $\mu(K[X])<k$, and by 1) $\mu_{*}(R)<k$.

Proposition 22. Let $R=K\left[X^{k}, a X^{l}\right]$ for some positive integers $k, l$ and some $a \in K\left[X^{k}\right] \backslash X^{k} K\left[X^{k}\right]$ such that $k>1,(k, l)=1$ and $X^{l} \notin R$ if $l<k$. Then 
1) $C=X^{(k-1)(l-1)} a^{k-1} K[X]$, and

2) $C_{M}$ is a maximally generated ideal of $R_{M}$ for each $M \in \operatorname{Ass}(C)$.

Proof. 1) Let $f=X^{(k-1)(l-1)} a^{k-1}$. By Proposition $21, \bar{R}=K[X]$. We will show that $f K[X] \subseteq R$. Since $K[X]=\left(1, X, \ldots, X^{k-1}\right) R$ it is enough to show that $f X^{i} \in R$ if $i \in\{0, \ldots, k-1\}$. Let $i \in\{0, \ldots, k-1\}$. As $(k, l)=1$ there is $\mu, \nu \in \mathbf{Z}$ such that $f X^{i}=X^{(k-1)(l-1)+i} a^{k-1}=X^{k \nu+l \mu} a^{k-1}$. Since $\mu=t k+j$ for some $j \in\{0, \ldots, k-1\}$, and $t \in \mathbf{Z}$ we have $k \nu+l \mu=k(\nu+l t)+l j$, and therefore we can assume that $0 \leq \mu \leq k-1$. Then $(k-1)(l-1)+i=$ $k \nu+l \mu$, and $(k-1-\mu) l+i+1=k \nu+k$. Thus $1 \leq k \nu+k$, and hence $0 \leq \nu$ whence $f X^{i} \in R$, and $f K[X] \subseteq R$. If $c \in R$ is such that $C=c K[X]$ then $c$ divides $f$ in $K[X]$. We have $i=k-1$ if and only if $(k-1-\mu) l=k \nu$, i.e. if and only if $\nu=0$ and $\mu=k-1$. Hence $a^{k-1}$ divides $c$. If $(k-1) \times$ $(l-1)-1=k \nu+l \mu$ then $(k-1-\mu) l=k(\nu+1)$ which is impossible. Hence $f X^{-1} \notin C$, and thus $c=f$.

2) If $M=X K[X] \cap R$ then $M_{M}=\left(X^{k}, X^{l}\right)$, and $C_{M}=X^{(k-1)(l-1)}$. $\left(1, X, \ldots, X^{\min \{k, l\}}\right)$. Thus $X^{\min \{k, l\}} C_{M}=M_{M} C_{M}$. If $M \in \operatorname{Ass}(C)$, and $a \in M$ then $M=\left(b, a X^{l}\right)$ for some $b \in K\left[X^{k}\right]$ such that $b$ divides $a$ in $K\left[X^{k}\right]$, and $C_{M}=a^{k-1}\left(1, X, \ldots, X^{k-1}\right)$. Hence $b C_{M}=M_{M} C_{M}$, and $C_{M}$ is maximally generated for each $M \in \operatorname{Ass}(C)$.

Proposition 23. Let $R=K\left[X^{k}, a X^{l}\right]$ for some positive integers $k, l$ and some $a \in K\left[X^{k}\right] \backslash X^{k} K\left[X^{k}\right]$ such that $k>1,(k, l)=1$, and $X^{l} \notin R$ if $l<k$. Let $\operatorname{Ass}(C)=\left\{M_{i}\right\}_{i=1}^{t}$, and let $M_{1}=X K[X] \cap R$.

1) Then $\mu\left(C_{M_{1}}\right)=\min \{k, l\}$, and if $a \notin K$ then $\mu\left(C_{M_{i}}\right)=\mu_{*}(R)=k$, for $i=2, \ldots, t$.

2) Let $I_{i}$ be the unique biggest $M_{i}$-primary ideal of $R$ such that $\mu\left(I_{i}\right)=\mu_{*}\left(R_{M_{i}}\right), i=1, \ldots, t$. Then $I_{i}=M_{i}^{\mu\left(C_{M_{i}}\right)-1}, i=1, \ldots, t$.

Proof. 1) Suppose $a \notin K$, let $i \in\{2, \ldots, t\}$ and let $S=R \backslash M_{i}$, where $M_{i}=\left(b, a X^{l}\right)$ for some $b \in K\left[X^{k}\right]$ such that $b$ divides $a$ in $K\left[X^{k}\right]$. By Proposition 22, $C_{M_{i}}=a^{k-1}\left(1, X, \ldots, X^{k-1}\right)$, and if $\mu\left(C_{M_{i}}\right)<k$ then $a^{k-1} X^{j}=$ $a^{k-1} \sum_{\nu=1, \nu \neq j}^{k-1} \frac{r_{\nu}}{s_{\nu}} X^{\nu}$ for some $j \in\{1, \ldots, k-1\}, r_{\nu} \in R$ and $s_{\nu} \in S$, such that $r_{\nu}=1$ or $b$ divides $r_{\nu}, \nu=1, \ldots, j-1, j+1, \ldots, k-1$. Let $s=\prod_{\nu=1, \nu j}^{k-1} s_{\nu}$. Then $s\left(X^{j}-\sum_{\nu, r_{\nu}=1} \frac{X^{\nu}}{s_{\nu}}\right)=s\left(\sum_{\nu, r_{\nu} \neq 1} \frac{r_{\nu}}{s_{\nu}} X^{\nu}\right)$, and $b$ divides the right side but not the left one which is impossible. Thus $\mu\left(C_{M_{i}}\right)=k$, and by Proposition 21, $\mu_{*}(R)=k$. Since $M_{1 M_{1}}=\left(X^{k}, X^{l}\right)$, and $C_{M_{1}}=X^{(k-1)(l-1)}\left(1, X, \ldots, X^{\min \{k, l\}}\right)$ we have $\mu\left(C_{M_{1}}\right)=\min \{k, l\}$.

2) We have that the embedding dimension emdim $R_{M_{i}}=\ell\left(M_{i M_{i}} / M_{i M_{i}}^{2}\right)=2$, $i=1, \ldots, t$, and by [3], Theorem $1, I_{i M_{i}}$ is the conductor of $R_{M_{i}}$ in its blowing up ring at $M_{i M_{i}}$. By [11], Proposition 1.8, $I_{i M_{i}}=M_{i_{M_{i}}}^{e\left(R_{M_{i}}\right)-1}$, and by [2], Proposition 4.8, $I_{i}=M_{i}{ }^{e\left(R_{M_{i}}\right)-1}, i=1, \ldots, t$ where $e\left(R_{M_{i}}\right)$ is the multiplicity of 
$R_{M_{i}}$. By Proposition 22, $C_{M_{i}}$ is maximally generated, and by definition and Remark 12, $e\left(R_{M_{i}}\right)=\mu\left(C_{M_{i}}\right), i=1, \ldots, t$.

By Proposition 23 and Theorems 11 and 18 the set of proper strong $\mu_{*}(R)$ generators of $R$ is completely determined when $R=K\left[X^{k}, a X^{l}\right]$.

Let $B$ be a primitive numerical semigroup generated by some positive integers $n_{1}, \ldots, n_{l}$. Then $\left(n_{1}, \ldots, n_{l}\right)=1$, and there is a least integer $g(B)$ such that $g(B)+i \in B$ for $i=1,2, \ldots$ The integer $g(B)$ is called the Frobenius number of $B$.

Proposition 24. Let $n_{1}, \ldots, n_{t}$ be positive integers such that $\left(n_{1}, \ldots, n_{t}\right)=1$, and let $K[X ; B]=K\left[X^{n_{1}}, \ldots, X^{n_{t}}\right]$. Let $R$ be a ring such that $K[X ; B] \subseteq R \subseteq$ $K[X]$ where $B$ is maximal such that $K[X ; B] \subseteq R$. Let $N=g(B)+1$, and $M=X K[X] \cap R$. Then

1) $C=X^{N} K[X]$,

2) $\mu_{*}(R)=\min v(M)$, and if $\min v(M)=1$ then $R=K[X]$.

Proof. 1) By Proposition 21, $\bar{R}=K[X]$. As $X^{N} K[X] \subseteq C$, and $X^{N-1} \notin R$ we have $C=X^{N} K[X]$.

2) Let $\min v(M)=l$, and let $f$ be a polynomial in $M$ such that $v(f)=l$. Let $m \in \mathrm{N}$ be minimal such that $X^{m} K[X] \subseteq\left(X^{N}, \ldots, X^{N+l-1}\right) R$. If $m>N$ then let $i \in\{0, \ldots, l-1\}$ and $j \geq 0$ be such that $N+j l+i=m-1$. Hence $X^{N+i} f^{j}$ and $X^{N+i}\left(f^{j}-a X^{j l}\right)$ belong to $\left(X^{N}, \ldots, X^{N+l-1}\right)$ for some $a \in K$ such that $f^{j}-a X^{j l}=0$ or $v\left(f^{j}-a X^{j l}\right)>j l$. Thus $X^{N+i+j l}=X^{m-1} \in\left(X^{N}, \ldots\right.$, $\left.X^{N+l-1}\right)$ which is a contradiction. Hence $m=N$, and $\mu(C) \leq l$. Since $C_{M} / M C_{M}=\left(\bar{X}^{N}, \bar{X}^{N+1}, \ldots, \bar{X}^{N+l-1}\right)$ where $\bar{X}$ is the image of $X$ in $R_{M} / M R_{M}$, and $\bar{X}^{N}, \bar{X}^{N+1}, \ldots, \bar{X}^{N+l-1}$ are linearly independent in the $R_{M} / M R_{M}$-vector space we have $\mu(C)=\mu\left(C_{M}\right)=l$. By Theorem 15, $\mu_{*}(R)=l$. If $\min v(M)=1$ then $R$ is a PID, and hence integrally closed. Thus $R=K[X]$.

Remark 25. By Proposition 24, $C$ is primary for $M=X K[X] \cap R$, and by Theorem 15, $\mu_{*}\left(R_{M}\right)=\mu_{*}(R)$. We have $K[X]_{M} / X K[X]_{M} \simeq R_{M} / M_{M}$, and $K[X]_{M}$ is local. By Remarks 12 and 17 the biggest $M$-primary ideal $I$ such that $\mu(I)=\mu_{*}(R)$ can be determined, and by Theorems 11 and 18 the set of proper strong $\mu_{*}(R)$-generators in $R$ is determined. If moreover $K$ is infinite then $R_{M} / M_{M}$ is infinite, and by Remark 12 there is a superficial element in $R_{M}$.

AcKnowdgements. I am grateful to my supervisors R. Fröberg, C. Gottlieb and C. Löfwall for their advice and comments during the work with this paper. 


\section{REFERENCES}

1. D.D. Anderson and L.A. Mahaney, Generating sets for ideals, Comment. Math. Univ. St. Paul. 37 (2) (1988), 173-177.

2. M.F. Atiyah and I.G. MacDonald, Introduction to Commutative Algebra, Addison-Wesley Publishing Company, 1969.

3. V. Barucci and K. Pettersson, On the biggest maximally generated ideal as the conductor in the blowing up ring, Manuscripta Math. 88 (1995), 457-466.

4. H. Bass, Torsion free and projective modules, Trans. Amer. Math. Soc. 102 (1962), 319-327.

5. S.T. Chapman and N.H. Vaughan, A theorem on generating ideals in certain semigroup rings, Boll. Un. Mat. Ital. A. 7 (1991), 41-49.

6. I.S. Cohen, Commutative rings with restricted minimum condition, Duke Math. J. 17 (1950), $27-42$.

7. E.D. Davis and A.V. Geramita, Efficient generation of maximal ideals in polynomial rings, Trans. Amer. Math. Soc. 231 (1977), 497-505.

8. O. Forster, Über die Anzahl der Erzeugenden eines Ideals in einem Noetherschen Ring, Math. Z. 84 (1964), 80-87.

9. C. Gottlieb, On generators of ideals in one-dimensional local rings, Comm. Algebra 21 (2) (1993), 421-425.

10. H. Matsumura, Commutative Ring Theory, Cambridge Univ. Press, 1992.

11. F. Orecchia and I. Ramella, The conductor of one-dimensional Gorenstein rings in their blowing up, Manuscripta Math. 68 (1990), 1-7.

12. K. Pettersson, Strong n-generators in some one-dimensional domains, Comm. Algebra 22 (8) (1994), 2941-2953.

13. I. Stewart, Galois Theory, Chapman and Hall, 1973.

14. O. Zariski and P. Samuel, Commutative Algebra Vol. 2, Springer, 1976.

DEPARTMENT OF MATHEMATICS

UNIVERSITY OF STOCKHOLM

S-10691 STOCKHOLM

SWEDEN 